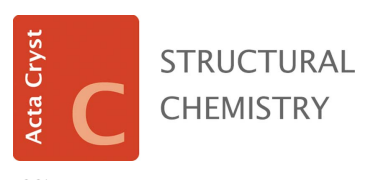

ISSN 2053-2296

\section{New node on the block for organic solid-state chemists: rtct-tetrakis(pyridin-4-yl)cyclobutane}

Eric W. Reinheimer*

Rigaku Americas Corporation, 9009 New Trails Drive, The Woodlands, TX 77381, USA. *Correspondence e-mail: eric.reinheimer@rigaku.com

As chemists and crystallographers, growing a crystal and obtaining the structure of a synthesized molecule is often considered the dénouement of a sometimes long and often arduous process. To peer into the atomic world and view the three-dimensional model of a molecule that might determine future research projects is the greatest reward for these interminable hours in the lab. While the molecules and the conclusions may outlast your graduate, postdoctoral, or professional career, are all syntheses and applications truly complete with that crystal? Is that the end or can more be done?

Seminal work by Schmidt and co-workers in 1971 signaled the advent of organic solidstate syntheses and demonstrated that even within a single crystal additional synthetic work was possible. In their report, Schmidt reported that trans-cinnamic acid and its derivatives were capable of undergoing a $[2+2]$ photodimerization to generate functionalized cyclobutanes post-crystallization (Schmidt, 1971). As a direct result of this observation, the authors ultimately proposed the 'Topochemical Postulate', which hypothesized that olefin-containing molecules within $4.2 \AA$ of one another can undergo a photodimerization in the solid state.

While cinnamic acid crystallizes in such a way that the olefin is rendered photoactive, a far greater number of olefin-containing molecules as a consequence of their crystal packing are photostable and are unable to undergo a photodimerization reaction. Pioneering research in crystal engineering has demonstrated that in order to perpetuate the onset of photoreactivity in these latter instances, the use of templates is required to align the reactant molecules in order to photoreact (Gan et al., 2018). Numerous supramolecular interactions, such as hydrogen and halogen bonding, argentophilic and aurophilic interactions, along with others, have proven to be reliable in the formation of reactive solids containing olefins that are photostable as a single-component solid. This templated approach to solid-state synthesis has yielded novel molecules (i.e. cyclophanes and ladderanes) whose yield is prohibitively low when prepared through a standard solution-based approach. The progress of the solid-state syntheses can be followed by ${ }^{1} \mathrm{H}$ NMR as the disappearance of olefinic $\mathrm{H}$ atoms coupled with the concomitant appearance of $\mathrm{H}$ atoms from cyclobutane moieties signals the onset of photoproduct formation (Campillo-Alvarado et al., 2019; Chanthapally et al., 2014; MacGillivray et al., 2000; Pahari et al., 2019; Sezer et al., 2017; Volodin et al., 2018).

Even though the solid-state syntheses of functionalized cyclobutanes have yielded some very aesthetically appealing molecules and crystal structures, further synthetic efforts utilizing these systems can be undertaken with the rational design of highlyconnected networks. Careful consideration of both the nodes and linkers is essential given their direct influence on the topology of the network (Jiang et al., 2018). Functionalized cyclobutane molecules generated using the $[2+2]$ photodimerization reaction have been successfully incorporated as nodes within these connected networks. A survey of the literature has demonstrated that the most studied photoproduct within these networks is the $r c t t$-isomer of tetrakis(pyridin-4-yl)cyclobutane (rctt-TPCB), which has functioned as a node in both metal-based and molecular networks (Li et al., 2014; Blake et al., 1997; Oburn et al., 2020; Baldrighi et al., 2010). While the rctt-isomer of tetrakis(pyridin-4-yl)cyclobutane is the most-often utilized, a second isomer (rtct-TPCB) is known and considered to be more thermodynamically stable. The $\mathrm{H}_{2} \mathrm{SO}_{4}$-catalyzed procedure to prepare the $r t c t$-isomer from its $r c t$ homologue in dimethyl sulfoxide (DMSO) was first outlined in 2010 (Peedikakkal et al., 2010); however, no single-crystal 


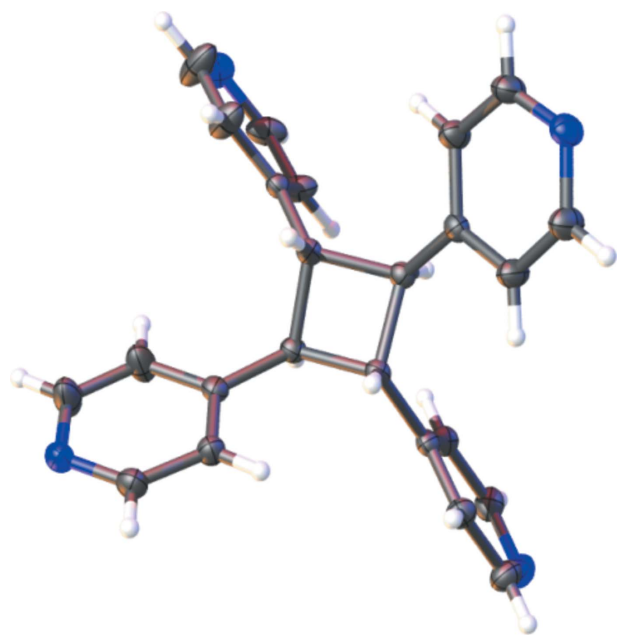

Figure 1

Rendering of the asymmetric unit of rtct-TPCB. Anisotropic displacement ellipsoids have been set at the $50 \%$ probability level.

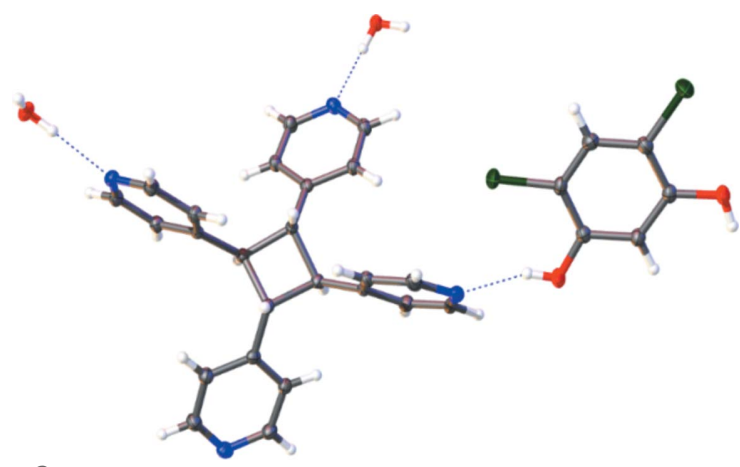

Figure 2

The functionalized cyclobutane $\boldsymbol{r t c t}$-TPCB functioning as a hydrogenbond-accepting three-connecting node by engaging in $\mathrm{O}-\mathrm{H} \cdots \mathrm{N}$ hydrogen bonding. Anisotropic displacement ellipsoids have been set at the $50 \%$ probability level.

structure of $\boldsymbol{r t c t}$-TPCB or a structure demonstrating that it could, like $\boldsymbol{r c t t}$-TPCB, be incorporated into a purely molecular network was reported.

Thanks to the recent report by the Groeneman research group, the lack of a structure for the rtct-TPCB isomer (Fig. 1), as well as structural proof that it can function as a node in a molecular network, has been clarified for the solid-state and crystal engineering communities (Santana et al., 2021). Within the extended structure, rtct-TPCB functions as a three-connecting node by accepting three $\mathrm{O}-\mathrm{H} \cdots \mathrm{N}$ hydrogen bonds with included water molecules and 4,6-dichlororesorcinol (Fig. 2). This type of packing arrangement for the node is required to form the ornate honeycomb topology (Fig. 3). Ultimately, it was found that the honeycomb network formed a three-dimensional network through the onset of $\mathrm{C}-\mathrm{H} \cdots \mathrm{N}$ hydrogen bonding between a $\mathrm{H}$ atom on the cyclobutane and the final pyridyl $\mathrm{N}$ atom on a neighboring $r$ tct-TPCB.

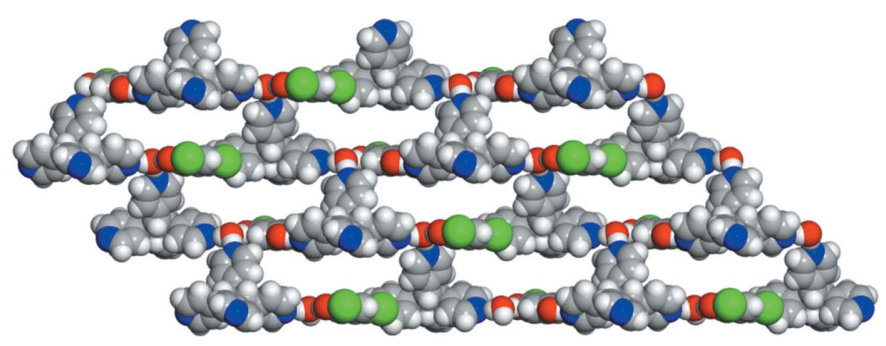

Figure 3

Extended view of the honeycomb network illustrating the observed channels.

Due to this highlighted work, we now know the symmetry and unit-cell parameters of the functionalized cyclobutane rtct-TPCB, as well as a multicomponent solid containing it. What other networks and topologies are possible? With the facile, acid-catalyzed procedure to generate other regioisomers, it is exciting to envision additional regioisomers of other functionalized cyclobutanes yet to be reported. The authors are to be applauded for finally addressing the paucity of information on this under-utilized isomer and shedding some light on it.

\section{References}

Baldrighi, M., Metrangolo, P., Meyer, F., Pilati, T., Proserpio, D., Resnati, G. \& Terraneo, G. (2010). J. Fluor. Chem. 131, 12181224.

Blake, A. J., Champness, N. R., Chung, S. S. M., Li, W.-S. \& Schröder, M. (1997). Chem. Commun. pp. 1675-1676.

Campillo-Alvarado, G., Li, C., Swenson, D. C. \& MacGillivray, L. R. (2019). Cryst. Growth Des. 19, 2511-2518.

Chanthapally, A., Oh, W. T. \& Vittal, J. J. (2014). Chem. Commun. 50, 451-453.

Gan, M.-M., Yu, J.-G., Wang, Y. Y. \& Han, Y.-F. (2018). Cryst. Growth Des. 18, 553-565.

Jiang, H., Jia, J., Shkurenko, A., Chen, Z., Adil, K., Belmabkhout, Y., Weselinski, L. J., Assen, A. H., Xue, D.-X., O'Keeffe, M. \& Eddaoudi, M. (2018). J. Am. Chem. Soc. 140, 8858-8867.

Li, D.-X., Ni, C.-Y., Chen, M.-M., Dai, M., Zhang, W.-H., Yan, W.-Y., Qi, H.-X., Ren, Z.-G. \& Lang, J.-P. (2014). CrystEngComm, 16, 2158-2167.

MacGillivray, L. R., Reid, J. L. \& Ripmeester, J. A. (2000). J. Am. Chem. Soc. 122, 7817-7818.

Oburn, S. M., Santana, C. L., Elacqua, E. \& Groeneman, R. H. (2020). CrystEngComm, 22, 4349-4352.

Pahari, G., Bhattacharya, B., Reddy, C. M. \& Ghoshal, D. (2019). Chem. Commun. 55, 12515-12518.

Peedikakkal, A. M. P., Peh, C. S. Y., Koh, L. L. \& Vittal, J. J. (2010). Inorg. Chem. 49, 6775-6777.

Santana, C. L., Battle, J. D., Unruh, D. K. \& Groeneman, R. H. (2021). Acta Cryst. C77, 111-115.

Schmidt, G. M. J. (1971). Pure Appl. Chem. 27, 647-678.

Sezer, G. G., Yeşilel, O. Z. \& Büyükgüngör, O. (2017). J. Mol. Struct. 1137, 562-568.

Volodin, A. D., Korlyukov, A. A., Zorina-Tikhonova, E. N., Chistyakov, A. S., Sidorov, A. A., Eremenko, I. L. \& Vologzhanina, A. V. (2018). Chem. Commun. 54, 13861-13864. 